\title{
EL SANEAMIENTO PREDIAL Y SU VALIDACIÓN DESDE LA ACTUACIÓN DE DIVERSOS ACTORES IMPLICADOS EN LA DINÁMICA TERRITORIAL DE LOS HUMEDALES DE LA CIUDAD DE BOGOTÁ, COLOMBIA
}

\author{
PROPERTY SANITATION AND ITS VALIDATION THROUGH \\ THE ACTIONS OF VARIOUS PLAYERS INVOLVED \\ IN THE TERRITORIAL DYNAMICS OF THE WETLANDS \\ OF THE CITY OF BOGOTÁ, COLOMBIA
}

${ }^{1}$ Gina Paola González Angarita
2Valeria Toro Casas
${ }^{3}$ Elkin Oswaldo Dorado Castillo

${ }^{1}$ Dr. Ordenación del Territorio y Medio Ambiente, Universidad Libre, Bogotá Colombia.

${ }^{2}$ Est.Ingenieria Ambiental, Universidad Libre, Bogotá Colombia.

${ }^{3}$ Est.Ingenieria Ambiental, Universidad Libre, Bogotá Colombia.

\footnotetext{
${ }^{1}$ ginap.gonzaleza@unilibre.edu.co

${ }^{2}$ valeria-toroc@unilibre.edu.co

${ }^{3}$ elkino-doradoc@unilibre.edu.co
}

\section{RESUMEN}

Esta investigación estudia el saneamiento predial (recuperación de terrenos) a partir de las percepciones de actores involucrados con los humedales de Bogotá. El trabajo se inicia con un diagnóstico de los conflictos en los usos de los suelos durante el 2004, que involucra las partes interesadas, es decir los terrenos que se encuentran en posesión de propietarios externos a la gestión de los humedales, para determinar el grado de afectación en toda su extensión. El enfoque metodológico utiliza el análisis y gestión de entrevistas semiestructuradas, teniendo en cuenta el análisis narrativo de las partes interesadas, debido a sus conocimientos sobre la localización de zonas en conflicto y zonas de saneamiento predial. Las limitaciones de la investigación se presentan por la falta de conocimiento de las partes interesadas sobre las gestiones de saneamiento predial adelantadas en la zona de estudio. En síntesis, los resultados se presentan mediante cartografía social y estos revelaron que existe un $30 \%$ de zonas en conflicto y tan solo el $16 \%$ corresponde a la adquisición de terrenos para la conservación de humedales. Este análisis contribuye a una mejor compresión de la problemática actual sobre los usos de los suelos y sus necesidades de gestión, con la 
expectativa de que las autoridades competentes tomen en cuenta esta información.

Palabras clave saneamiento predial, conflicto de usos del suelo, cartografía social y humedales.

\section{ABSTRACT}

This research studies the property sanitation (land recovery) based on the perceptions of players involved in the Bogotá wetlands. The study begins with a diagnosis of conflicts in land use during 2004, which involves the stakeholders, that is, the lands that are in the possession of owners who do not have a management of wetlands, to determine their degree of affectation in the extension of these. The methodological approach uses the analysis and management of my structured interviews, which takes into account the narrative analysis of the interested parties for their knowledge on the location of conflict zones and areas of property sanitation. The limitations of the research are based on the lack of knowledge of the stakeholders on the management of property sanitation carried out in the study area. In summary, the results are presented by means of social mapping and these revealed that there is a $30 \%$ of conflict zones and only $16 \%$ corresponds to the acquisition of land for wetland conservation. This analysis contributes to a better understanding of the current problem of land uses and their management needs, with the expectation that they can be taken into consideration by the competent authorities.

Keywords: Property sanitation, land use conflict, social cartography and wetlands.

\section{Resumen gráfico}

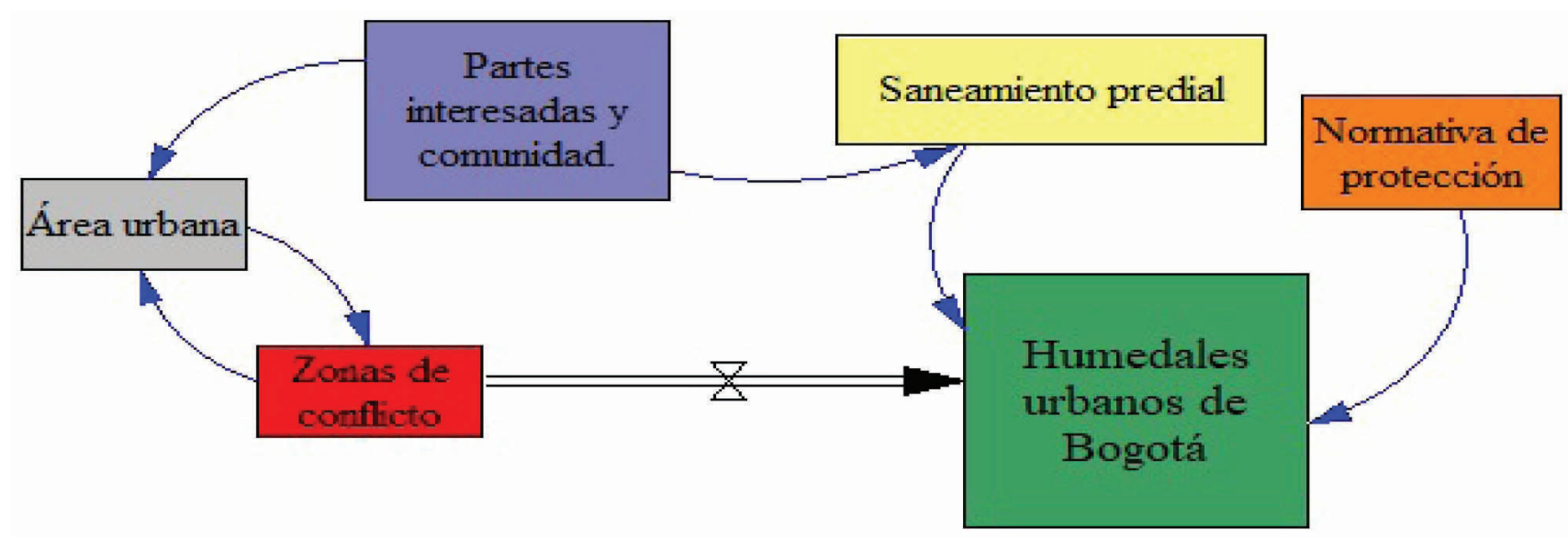

\section{INTRODUCCIÓN}

En los últimos 20 años, Colombia ha tenido un crecimiento urbano sin precedentes. Las principales ciudades del país han presentado un crecimiento predial superior al 3\% desde 1991 (Shlomo et al, 2018), lo que aumentará la presión sobre los recursos naturales. Esta situación ha causado efectos colaterales en los ecosistemas estratégicos en zonas urbanas, como es el caso de Bogotá: con un creciente ascenso poblacional, la ciudad se ha venido ocupando en una proporción de 58 habitantes por hectárea en el último decenio (Inostroza, Baur, \& Csaplovics, 2013).

Por lo tanto, las problemáticas actuales de Bogotá requieren con urgencia integrar nuevos 
lineamientos en sus planes territoriales. De acuerdo con Cerema (2015), las especificaciones urbanísticas deben integrar la protección de los medios húmedos. En ese sentido, en su plan de ordenamiento territorial, la ciudad de Bogotá ha venido adoptando la protección de los ecosistemas estratégicos mediante la política de humedales distritales (Alcadía mayor de Bogotá, 2004). La defensa, protección y recuperación de dichos ecosistemas se encuentra a cargo de organizaciones sociales, la Empresa de Acueducto y Alcantarillado de Bogotá (EAAB), la Secretaría Distrital de Medio Ambiente (SDA) y el Jardín Botánico de Bogotá (JBB), entre otras.

La normativa de protección de humedales de Bogotá se encuentra sustentada en la resolución 196 del 2006, que establece una guía técnica para la formulación de planes de manejo ambiental. Posteriormente, en el mismo año se estableció el decreto 062 para definir los mecanismos, lineamientos y directrices para la elaboración de Planes de Manejo Ambiental. La aplicación de la normativa incluye la protección de humedales desde el saneamiento predial, este se fundamenta con el programa de gestión de Bogotá-Colombia, que busca la adquisición del terreno para la restauración de los humedales, así sus actividades requieran la compra de zonas de propiedad privada, la eliminación de asentamientos ilegales y la reubicación de las personas instaladas en ellas (Escalona y González, 2016).

A pesar de la normativa de protección de humedales, en la actualidad son diversos los conflictos de uso del suelo que aquejan a los humedales de Bogotá, puesto que muchos de ellos son ocasionados por la lenta gestión en los procesos de saneamiento predial y su escasa inversión económica desde los planes de gobierno.
De acuerdo con la EAAB (2013), las inversiones económicas en saneamiento predial se encuentran alrededor de USD 78 276,54 para cubrir periodos de tiempo desde el 2005 hasta el 2013. De esta manera, se deduce que son pocos los recursos económicos, lo que significa que la adquisición de terrenos es muy baja, razón por la cual el $51 \%$ de los terrenos de los humedales son de propiedad privada (EAAB, 2017). Esta investigación busca analizar los procesos de gestión del saneamiento predial teniendo como referencia los conocimientos de los actores involucrados mediante cartografía social, entrevistas semiestructuradas y trabajo de campo.

\section{MATERIALES Y MÉTODOS}

\section{Área de estudio}

Las gestiones adelantadas en el saneamiento predial están dirigidas a doce humedales localizados en el occidente de la ciudad de Bogotá, que se encuentran emplazados en las localidades de Usaquén, Bosa, Kennedy, Fontibón, Engativá y Suba. Por lo tanto, limitan al oriente con los cerros orientales, la Calera y el pueblo de Choachí; al norte, con el municipio de Chía; $y$ al sur con Soacha.

En la Figura 1 se observa la localización de los humedales. Al norte de la ciudad se localiza el Torca-Guaymaral; continuamente, por el noroccidente, se encuentran los humedales Córdoba, Conejera, Tibabuyes y Jaboque; seguidamente, en la zona central de Bogotá, se localizan los humedales Santa María del Lago, Capellanía, Meandro de Say; y finalmente, al suroriente, se emplazan los humedales Techo, Burro, Vaca, y Tibanica. En general la red de humedales limita al occidente con el río Bogotá. 


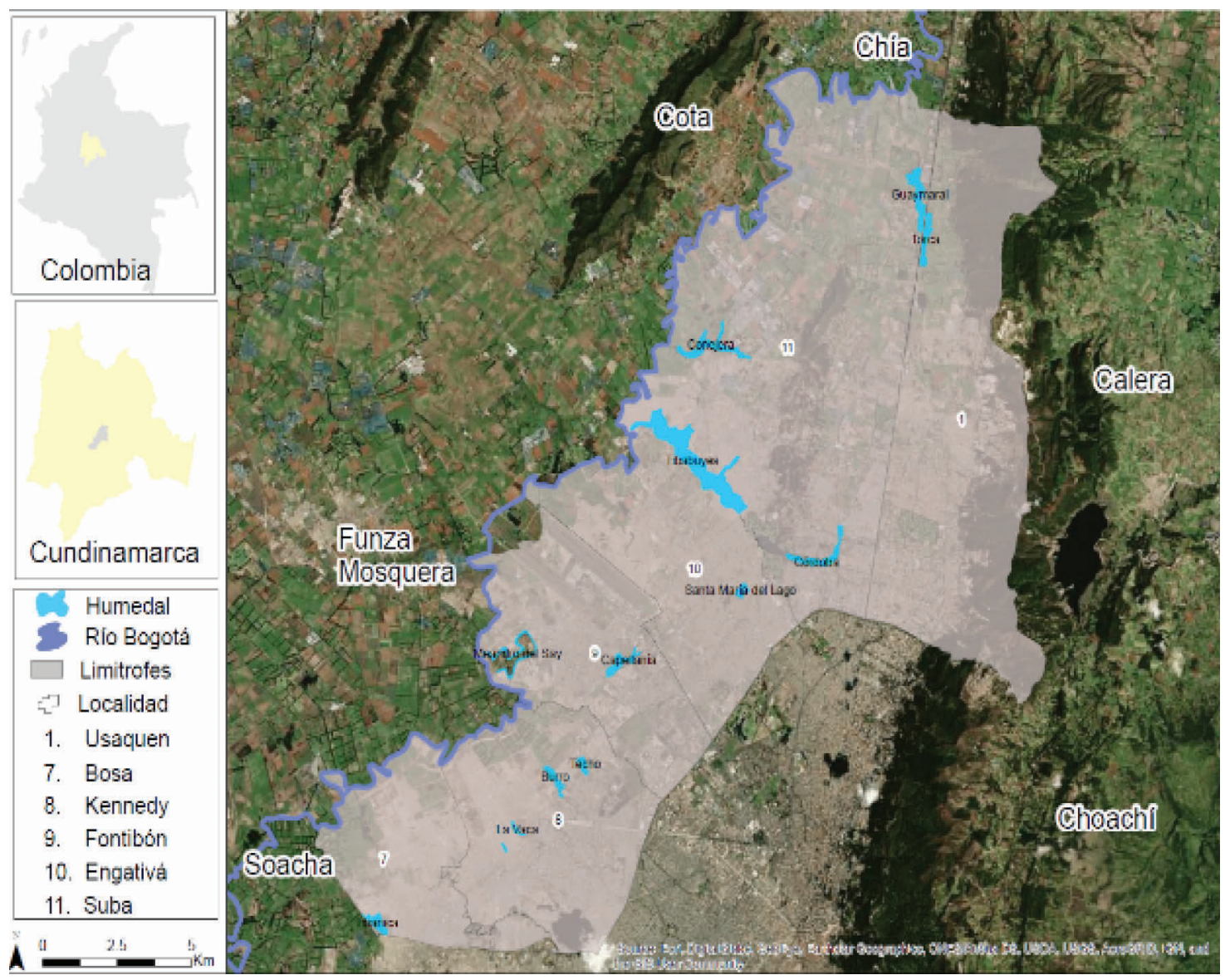

Figura 1. Localización de los humedales. Fuente: Elaboración propia.

\section{METODOLOGÍA}

Los fundamentos metodológicos de la presente investigación se derivan de técnicas exploratorias y descriptivas, cuyo objetivo es identificar el conflicto de usos del suelo a partir del trabajo de campo para la construcción de la cartografía social. Su elaboración requiere de realizar entrevistas semiestructuradas. En este caso, los factores más relevantes para analizar la gestión del saneamiento predial se consideran para identificar la situación actual de la superficie de los humedales.

\section{Entrevistas semiestructuradas}

Se emplearon entrevistas semiestructuradas debido a que esta técnica ayuda analizar las percepciones de los actores de las partes interesadas. Tal y como lo propone Ricart y Clarimont, (2016) quienes afirman que la técnica es de gran utilidad para hacerle frente a la complejidad de los conflictos de gestión de los recursos hídricos, puesto que muchas veces los enfoques técnicos no son suficientes. Por esto, la expresión "partes interesadas" hace referencia a un grupo de personas que comparten un interés común (Grimble y Wellard, 1997). En ese orden de ideas, se seleccionaron los actores con base en sus conocimientos en la gestión del saneamiento predial en los humedales de Bogotá. 
De esta manera los actores más representativos son líderes comunitarios y funcionarios públicos de la SDA, el JBB, las alcaldías locales de la ciudad y la EAAB. Debido a que, aunque existan muchos actores, pocos son los que tienen conocimiento acerca del saneamiento predial de humedales, la muestra se redujo ( $n=14)$. Según Bryson, (2004) el desarrollo de las entrevistas debe involucrar solo a los actores más importantes, para entender mejor sus problemáticas y encontrar soluciones. Por esto, para la selección de actores se requirió de la participación en diversos comités de las alcaldías locales, visitas de campo a los humedales y revisión de los participantes en los planes de manejo ambiental. En la Tabla 1 se identifican los principales actores implicados en la interacción de la dinámica de los humedales. Las entrevistas fueron realizadas en el año 2015 y se requirió de 12 meses para su organización, los entrevistados respondieron las preguntas en forma presencial y por correo electrónico. Se hizo énfasis en la construcción de cartografía social para identificar la Adquisición predial o zonas de recuperación en los humedales de Bogotá. La lista de preguntas se dividió en dos partes: *Datos identificativos y adquisición predial

Tabla 1. Listado y función de las partes interesadas en el saneamiento predial de los humedales de Bogotá.

\begin{tabular}{|c|c|c|c|}
\hline Partes interesadas & Acrónimo & Grupo & Función \\
\hline $\begin{array}{l}\text { Red intercultural } \\
\text { canto al agua }\end{array}$ & RICA & Privado & $\begin{array}{l}\text { Movimiento ambiental y cultural que busca la reflexión } \\
\text { en la población }\end{array}$ \\
\hline $\begin{array}{l}\text { Fundación Banco } \\
\text { de semillas }\end{array}$ & FBS & Privado & $\begin{array}{l}\text { Gestión y concientización de la protección de los } \\
\text { humedales en la localidad de Kennedy }\end{array}$ \\
\hline $\begin{array}{l}\text { Empresa } \\
\text { de acueducto } \\
\text { y alcantarillado }\end{array}$ & EAA & Público & $\begin{array}{l}\text { Presta servicios de acueducto y alcantarillado y gestiona } \\
\text { el tratamiento de agua residual de Bogotá. }\end{array}$ \\
\hline Fundación Funcoajz & $\mathrm{FF}$ & Privado & $\begin{array}{l}\text { Grupo de ciudadanos que buscan la gestión ambiental } \\
\text { de los humedales de la localidad de Fontibón. }\end{array}$ \\
\hline Jardín Botánico & $\mathrm{JB}$ & Público & Manejo, gestión e investigación del arbolado de Bogotá. \\
\hline $\begin{array}{l}\text { Mesa ambiental } \\
\text { de Fontibón }\end{array}$ & MAF & Mixto & $\begin{array}{l}\text { Grupo de ciudadanos y funcionarios públicos de la alcaldía } \\
\text { local que buscan la gestión ambiental de los humedales } \\
\text { de la localidad de Fontibón. }\end{array}$ \\
\hline $\begin{array}{l}\text { Comité ambiental } \\
\text { de Suba }\end{array}$ & CAS & Mixto & $\begin{array}{l}\text { Grupo de ciudadanos y funcionarios públicos de la alcaldía } \\
\text { local que buscan la gestión ambiental de los humedales } \\
\text { de la localidad de Suba. }\end{array}$ \\
\hline $\begin{array}{l}\text { Comité ambiental } \\
\text { de Kennedy }\end{array}$ & CAK & Mixto & \\
\hline $\begin{array}{l}\text { Comité ambiental } \\
\text { de Engativá }\end{array}$ & CAE & Mixto & $\begin{array}{l}\text { Grupo de ciudadanos y funcionarios públicos de la alcaldía } \\
\text { local que buscan la gestión ambiental de los humedales } \\
\text { de la localidad de Engativá. }\end{array}$ \\
\hline
\end{tabular}

Fuente: elaboración propia a partir de las entrevistas semiestructuradas.

El desarrollo metodológico para las preguntas abiertas se realiza mediante un análisis de las narrativas, lo que implica analizar la descripción gruesa de las descripciones aportadas por el entrevistado (Lejano et al, 2019). En ese sentido se hace una caracterización 
general de las contribuciones de las partes interesadas con el fin de identificar la parte gruesa de las narrativas. Por ejemplo, en la siguiente narrativa, la entrevistada habla de dos temas muy importantes, en los que hace alusión a los predios por reasentamiento:

"Considera que es necesario terminar de adquirir predios bajo la firma de reasentamientos $[\ldots]^{\prime \prime}$

Después, introduce las temáticas de los empleos que se generan por efectos en la gestión en los humedales:

"[...] en el número de empleados de mantenimiento es la misma persona la que hace disposición final de residuos sólidos, la entrevistada hace la pregunta si cilos canales cuentan cómo saneamiento? dice que no es necesario un coordinador pedagógico y el biólogo debe estar permanente en el humedal; Manejar pasantes: Biología, Guías ambientales del SENA".

Por esto, la tarea analítica permite construir narrativas para cada una de las opciones

\section{Cartografía social y trabajo de campo}

La cartografía social se alimenta de las preguntas abiertas, como es el caso de la pregunta ¿Que predios habría que adquirir en su opinión? En esta fase, los entrevistados hacen algunas aportaciones como por ejemplo en el humedal Tibabuyes comprar terrenos del tercio medio y bajo, luego el entrevistado localiza las zonas en la cartografía. Tal y como se observa en la Tabla 2.

Tabla 2. Cartografía social y zonas reseñadas para recuperación de predios.

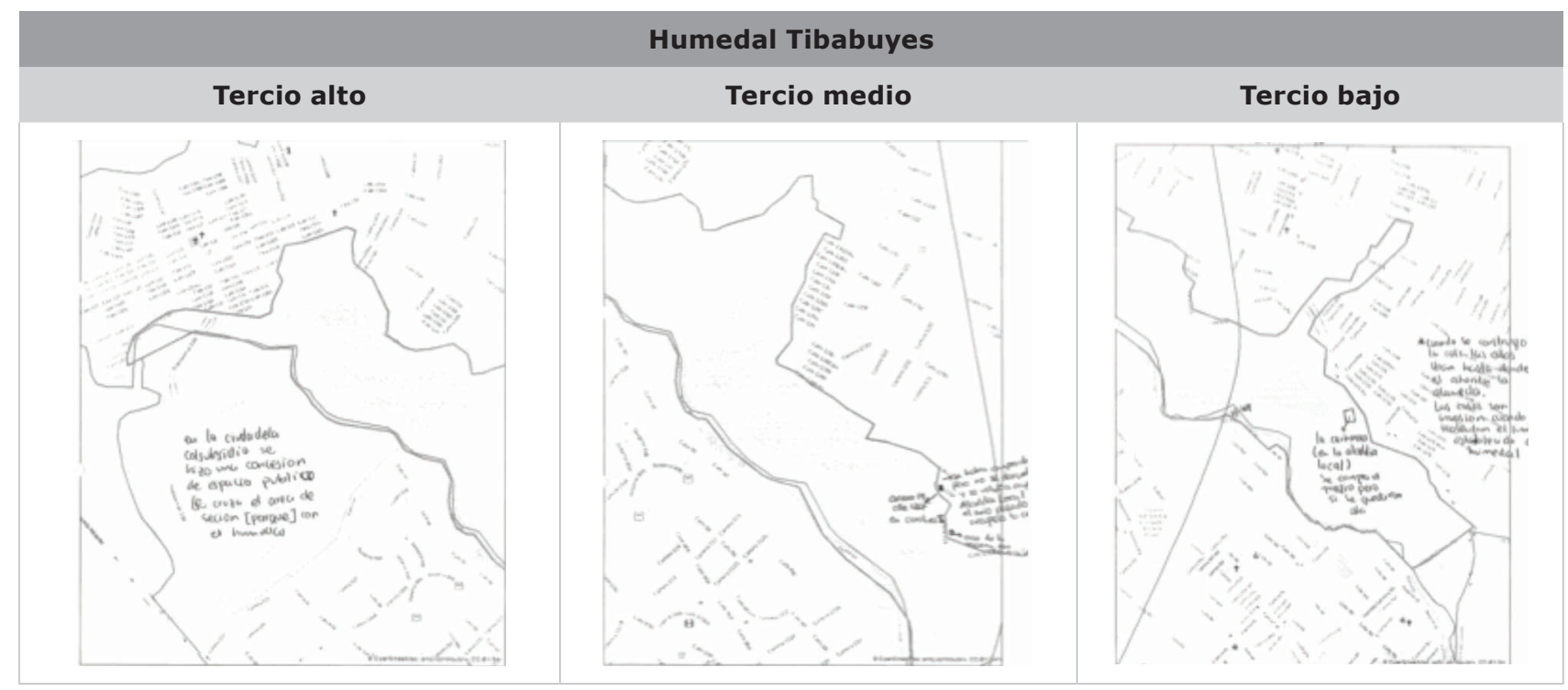

Fuente: elaboración propia a partir de las entrevistas realizadas.

A continuación, se procesó la geo especialización mediante el software ArcGis, considerando que los datos geoespaciales son un determinante, para identificar la superficie de las áreas en conflicto en el año 2004, primero del periodo estudiado, se ha continuado el siguiente procedimiento:

*Buscar en la página web del IDECA (http:// www.ideca.gov.co/es/servicios/mapa-de-referencia) mapas y cartografía de urbanización, vías, ciclo rutas y corrientes de agua. 
*Cargar los datos georreferenciados en un Sistema de Información Geográfica.

* Realizar un intersecto con los mapas de urbanización y los humedales para identificar las zonas en conflicto del año 2004.

* Validar la información geoespacial con la ortofoto del año 2004.

Después, en el año 2015 se realizó el mismo procedimiento. Sin embargo, la información se complementó con trabajo de campo, el cual consistía en la georreferenciación de los límites del humedal y las zonas de conflicto con la ayuda de un GPS Garmin etrex-30x. Posteriormente la información se sistematiza por medio de la herramienta Arc toolbox_ Conversion tools _ From GPS (Franco, 2011). Seguidamente, para la identificación de áreas recuperadas o saneamiento predial y zonas en conflicto en los humedales, se integran los actores sociales. Según Velez et al (2012), la identificación de zonas en conflicto en el recurso hídrico es una técnica propicia para conocer la situación real del área de estudio. De igual manera Aparacio et al (2018) identifican las zonas en conflicto interactuando con la comunidad para la identificación de conflicto de usos del suelo en los humedales Meandro del Say y Santa María del Lago de Bogotá. Así pues, la construcción de la cartografía social juega un papel importante en esta investigación para identificar el saneamiento predial y sus conflictos entorno a la red de humedales de Bogotá. Como ejemplo de la cartografía social aplicada en los humedales se aporta los mapas de los humedales Burro, Techo y Capellanía del año 2004 y 2015.

En las Figuras 2 y 3 se identifican los predios privados, asentamientos humanos y zonas de parqueadero que existían a finales del año 2004 en el humedal el Burro. Después, para la construcción cartográfica del año 201,5 se representa el saneamiento predial con la ayuda de la comunidad; entre ellos se identifican el Comité Ambiental local de Kennedy (CAK) y la Fundación Banco de Semillas (FBS), quienes identificaron los predios Ortero San Francisco y Senderos de Castilla como áreas recuperadas por efectos del saneamiento predial.

Además, en el trabajo de campo se observó un cambio en los usos de los suelos, puesto que desaparecieron algunos asentamientos ilegales $y$, en cambio, surgieron edificaciones al sur del humedal. Aunque son muchas las gestiones adelantadas en el humedal el Burro, las instituciones ambientales deben buscar estrategias para mitigar los efectos que causa la fragmentación vial ocasionada por la Avenida Ciudad de Cali. Asimismo, se debe promover la adquisición de predios privados, puesto que 


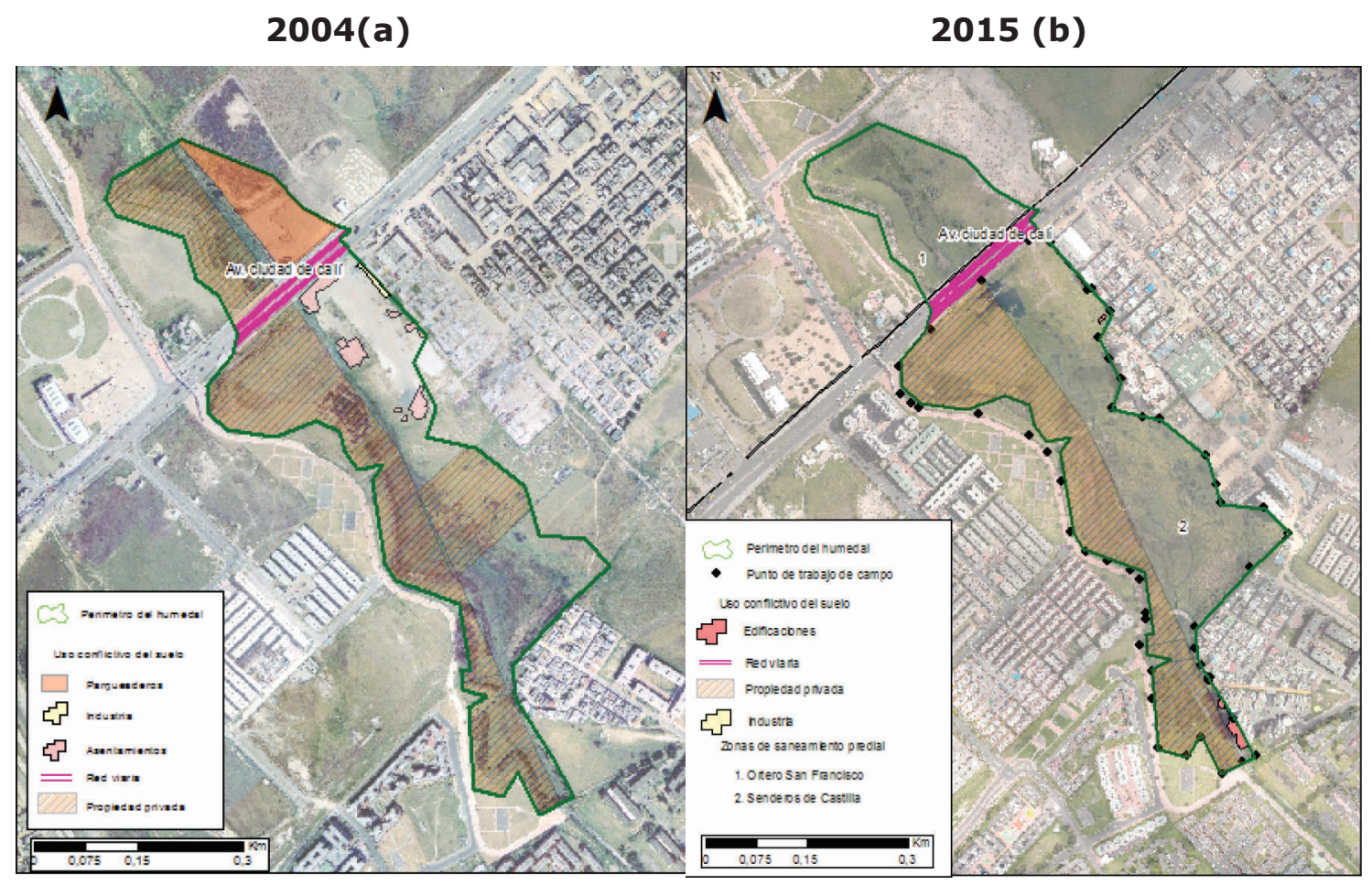

Figura 2. Delimitación de áreas en conflicto y la gestión del saneamiento predial humedal el Burro en 2004 (a)-2015(b).

Fuente: Elaboración propia a partir del trabajo de campo y las entrevistas semiestructuradas.

En cambio, la recuperación de la superficie del humedal de Techo se ha visto truncada por efectos del crecimiento urbano del barrio Lagos de Castilla, que invade el $35,85 \%$ de la superficie del humedal (González Angarita, 2018). La comparación entre los mapas de la figura 3 muestra una clara invasión debido a la malla vial y a los asentamientos ilegales que fueron reemplazados por edificaciones fortificadas.

Continuando con las comparaciones del humedal Capellanía, en la Figura 4 se identifica una clara reducción del área en conflicto con la recuperación de los predios de la urbanización Baleares III sector, Urbanización Tierra Firme y Cofradía III sector (Calderón, 2016). No obstante, los impactos sobre dicho humedal serán reiterativos por las directrices de Secretaría Distrital de Planeación (SDP) y el Instituto de Desarrollo Urbano, que tienen planeada la construcción de la Avenida Longitudinal de Occidente (ALO) (IDU, 2017). Si bien esta vía puede llegar afectar el 27,04\% de la superficie del humedal Capellanía, puesto que la pérdida de área disminuye las zonas verdes, zonas de preservación del humedal y su espejo de agua. 


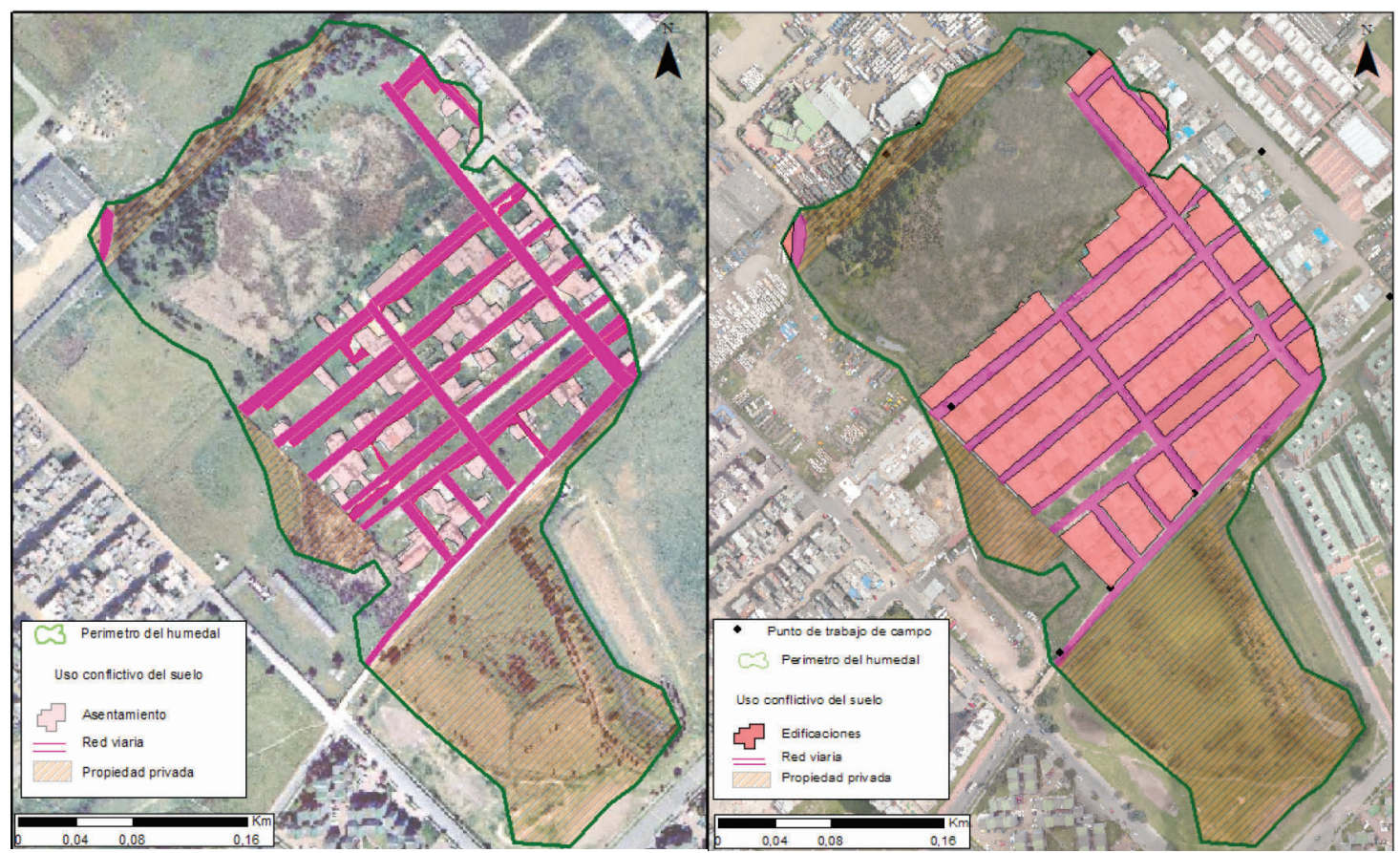

Figura 3. Delimitación de áreas en conflicto y la gestión del saneamiento predial humedal de Techo en 2004 (a)-2015(b). Fuente: Elaboración propia a partir del trabajo de campo y las entrevistas semiestructuradas.

2004

2015

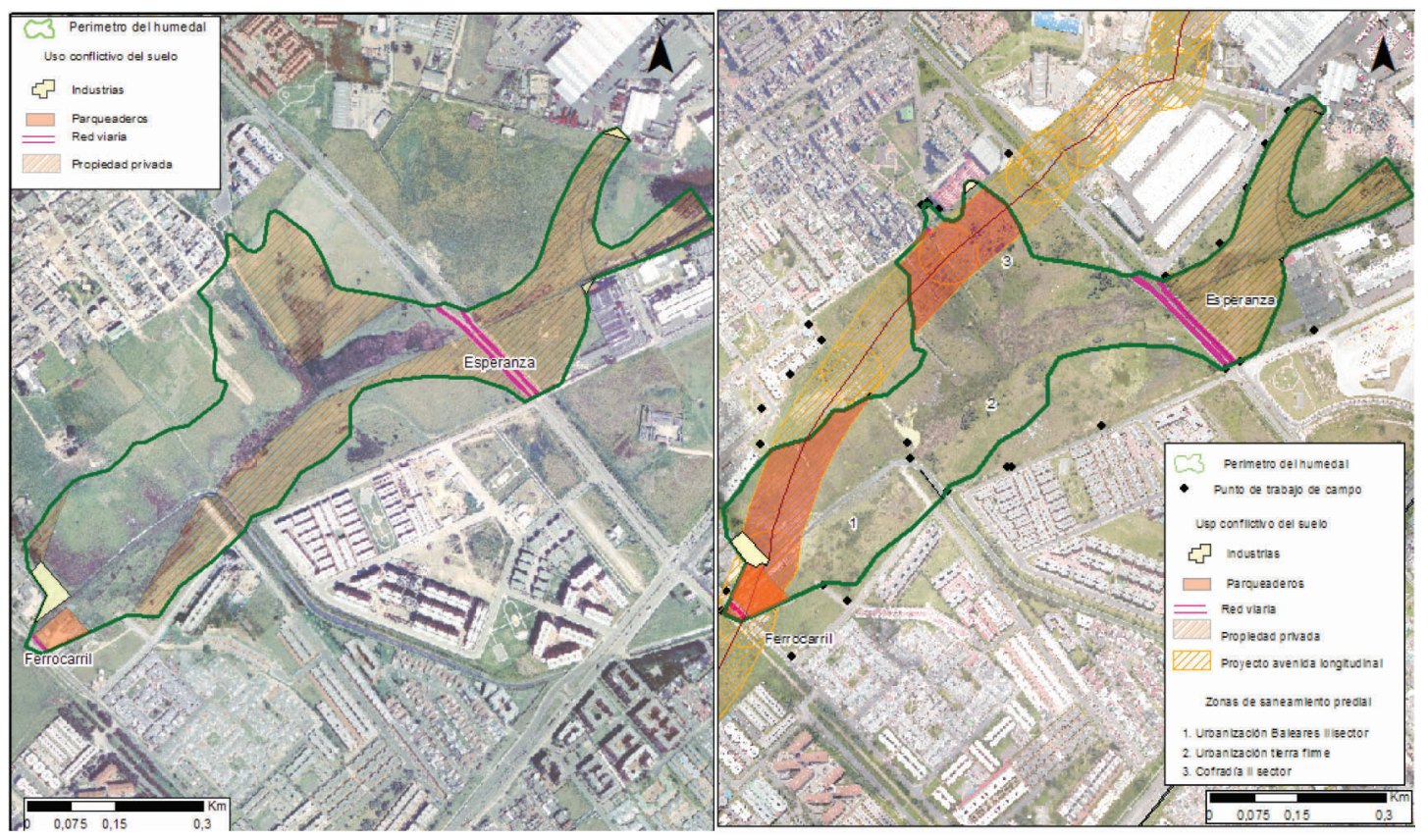

Figura 4. Delimitación de áreas en conflicto y la gestión del saneamiento predial del humedal Capellanía (2004 (a)-2015(b). Fuente: Elaboración propia a partir del trabajo de campo y las entrevistas semiestructuradas. 


\section{RESULTADOS}

De acuerdo con el desarrollo de las entrevistas realizadas, se encontró que los actores involucrados están directamente relacionados con las zonas de estudio. En ese sentido, el 22\% de las entrevistas corresponde a las localidades Engativá y Usaquén; el $21 \%$, a la localidad de Fontibón; el 14\% a las localidades de Bosa y Kennedy; y el 7\% a la localidad de Suba.

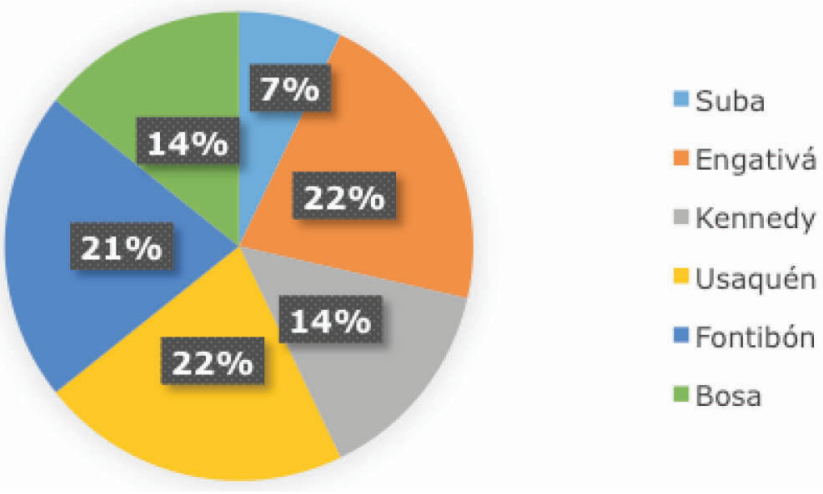

Figura 5. Desarrollo de entrevistas por localidad.

Fuente: Elaboración propia.

Con respecto a los datos recogidos en las entrevistas, que se representan en la Tabla 2, se ilustra la reducción de la superficie del área de estudio. Esto significa que, de las 674,06 hectáreas de la superficie de los humedales, tan solo el $16 \%$ corresponde a zonas recuperadas por las gestiones adelantadas desde el saneamiento predial. En cambio, las zonas en conflicto representan el 30\% de su extensión.

Tabla 2. Localización extensión y área recuperada en los humedales.

\begin{tabular}{|c|c|c|c|c|}
\hline Humedal & Localidad & $\begin{array}{l}\text { Extensión } \\
\text { (hectáreas) }\end{array}$ & $\begin{array}{l}\text { Área recuperada } \\
\text { (hectáreas) }\end{array}$ & $\begin{array}{l}\text { Área en conflicto } \\
\text { (hectáreas) }\end{array}$ \\
\hline Capellanía & Fontibón & 27,01 & 10,16 & 11,148 \\
\hline Torca-Guaymaral & Usaquén & 30,24 & 0 & 18,99 \\
\hline Tibabuyes & Suba & 222,58 & 46,55 & 36,63 \\
\hline Jaboque & Engativá & 147,88 & 15,79 & 63,06 \\
\hline Córdoba & Suba & 40,48 & 22,71 & 13,33 \\
\hline Conejera & Suba & 58,84 & 1,47 & 23,63 \\
\hline Techo & Kennedy & 11,66 & 0 & 11,23 \\
\hline Vaca & Kennedy & 7,96 & 4,17 & 1,53 \\
\hline El Burro & Kennedy & 18,82 & 4,05 & 7,06 \\
\hline Meandro del Say & Fontibón & 27,37 & 0 & 14,718 \\
\hline Santa María del Lago & Engativá & 10,85 & 0 & 1,061 \\
\hline Tibanica & Bosa & 70,37 & 4,07 & 2,89 \\
\hline Total & & 674,06 & 108,97 & 205,277 \\
\hline
\end{tabular}

Fuente: Elaboración propia. 
De acuerdo con los registros obtenidos en las entrevistas, se identificó que el saneamiento predial ejecutado en los humedales tiene mayor adquisición predial en los siguientes ecosistemas: Córdoba (21,8\%), Jaboque (14.3\%), Capellanía (9,56\%), Vaca $(4,17 \%)$. Además, los humedales que tuvieron una adquisición de predios mediana fueron entre el $1,33 \%$ y el $3,8 \%$ y entre ellos se identificó Tibabuyes (3,8 \%), Burro (3,7\%), Tibanica $(3,68 \%)$ y Conejera $(1,33 \%)$. Por último, el registro de los humedales que no tienen adquisición predial son los humedales de Techo, Meandro del Say, Torca-Guaymaral y Santa maría del Lago. En la Figura 5 se ilustra la clasificación de los humedales desde su mayor adquisición predial de predios privados hasta su menor adquisición.

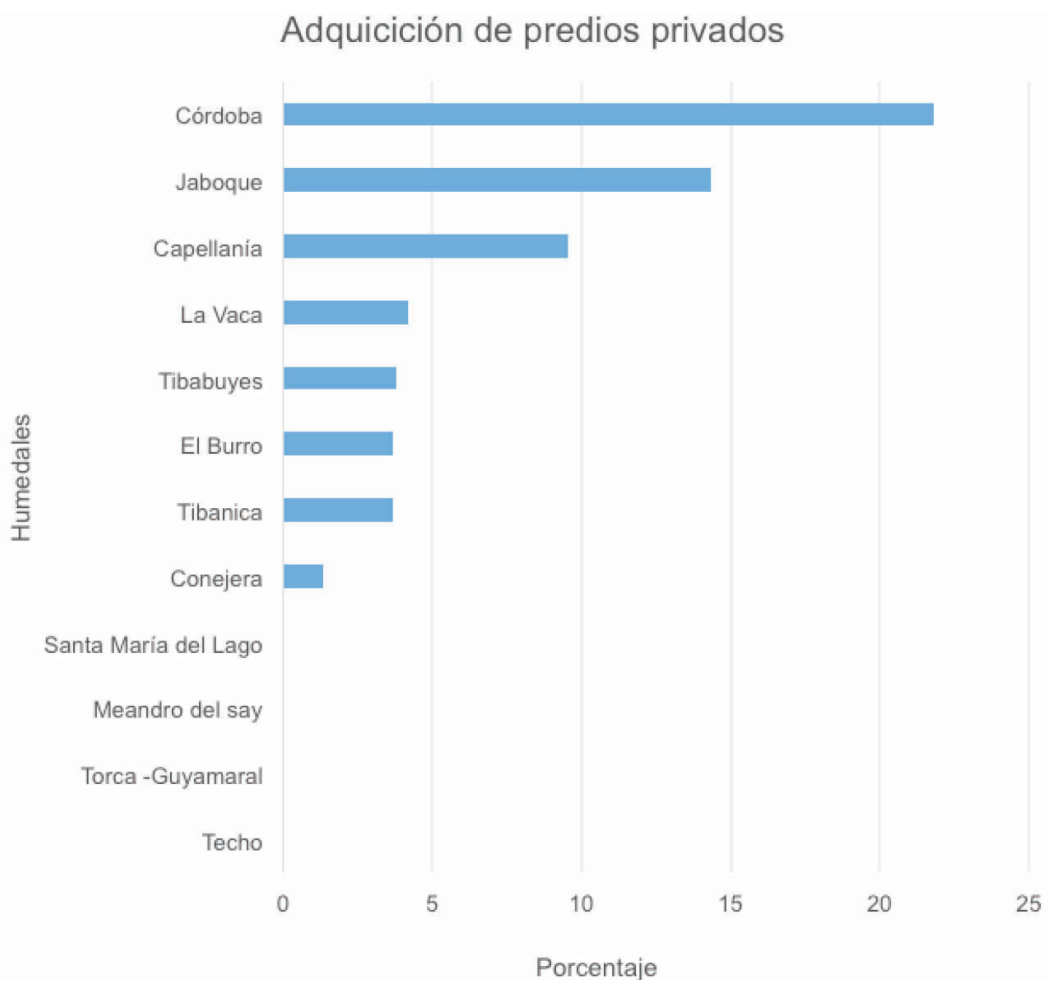

Figura 5. Resultados obtenidos en las entrevistas sobre la adquisición de predios privados y su clasificación por humedales.

Fuente: Elaboración propia.

\section{DISCUSIÓN Y CONCLUSIONES}

Con base en los resultados obtenidos en la presente investigación, se encontraron ciertos ámbitos por considerar. El primero indica que no es suficiente enfrentar los conflictos socio ambientales y desafíos de la gestión de humedales a partir de enfoques técnicos(Ricart \& Clarimont, 2016), puesto que los resultados indican que involucrar las partes interesadas, es decir los actores y expertos locales es un elemento clave para identificar las gestiones adelantadas desde el saneamiento predial en los humedales de Bogotá, puesto que el acceso a la información en las instituciones gubernamentales es impreciso debido a la falta de indicadores geoespaciales que permitan hacer un seguimiento continuo en las gestiones adelantadas en la adquisición de terrenos en materia de humedales. Además, se debe garantizar la divulgación de los terrenos adquiridos en diferentes canales para que exista una retroalimentación entre las 
partes interesadas y así mejorar la protección de dichos ecosistemas.

Una segunda contribución de este estudio fue el análisis del conflicto de usos del suelo en las zonas limítrofes a los humedales. De acuerdo con (Aparacio et al, 2018) el conflicto de usos de los suelos es evidente en los humedales Meandro del Say y Santamaría del Lago por efectos de la expansión urbana e industrial. En tanto esta situación se ratifica con los resultados obtenidos en la presente investigación, puesto que dichos ecosistemas demandan mayor atención en los procesos de saneamiento predial. Si bien en los resultados obtenidos se identifica que los humedales de Techo y Torca- Guaymaral, requieren también la recuperación de terrenos para disminuir los conflictos de usos de los suelos y ampliar sus rondas hidráulicas. En efecto el deterioro o desaparición de dichos humedales puede aumentar los riesgos por inundación para las localidades Kennedy, Engativá, Fontibón y Usaquén en la ciudad de Bogotá. Así pues, esta investigación contribuye en el campo de la gestión del saneamiento predial en la red de humedales de Bogotá, puesto que su validación en campo y el desarrollo de entrevista semiestructuradas ayuda a esclarecer cuáles son los predios que deben ser adquiridos por el gobierno para evitar su pérdida de área.

Por tanto, las futuras investigaciones deberán dirigirse al análisis cronológico de las zonas de inundación para planificar las zonas de expansión de la ciudad de Bogotá, sin tener que afectar las zonas de manejo y preservación ambiental en humedales. De igual manera, es necesario concienciar a las instituciones gubernamentales de la ciudad de Bogotá acerca de la funcionalidad que cumplen los humedales para el control de inundaciones. Otra línea de investigación futura que contribuya en la disminución del conflicto de usos del suelo se fundamenta con la implementación de Infraestructura verde en el contorno de los humedales, con el fin de ampliar las áreas naturales de los humedales.

En términos generales, el crecimiento del área urbana es una de las causas principales de la pérdida de zonas de manejo y preservación ambiental en los humedales, puesto que las dinámicas de la urbanización de Bogotá inician con procesos que impermeabilizan los suelos y aumentan la pérdida de superficie de los humedales.

\section{AGRADECIMIENTOS}

Agradecemos al centro de investigaciones de la Universidad libre por la financiación del proyecto de investigación "Evaluación de comparativa de los humedales de Bogotá" $N^{\circ} 11030136$, puesto que facilito el trabajo de campo con los estudiantes del programa de ingeniería ambiental. Asimismo, los reconocimientos se extienden a la doctora Ana Escalona de la universidad de Zaragoza, quien hizo aportes importantes en el desarrollo de la presente investigación. Finamente agradecemos a la beca de doctorado del Banco Santander de la Universidad de Zaragoza y a la beca de la Alianza del Pacifico.

\section{LITERATURA CITADA}

Aparacio Rodríguez, V., Avendaño Herrera, L., González Angarita, G., \& Peña Angulo, D. (2018). Análisis geoespacial de la dinámica de los humedales Meandro del Say y Santa María del Lago: Conflictos de usos del suelo y su pérdida de área. Avances, 131-149. doi:0.18041/17944953/avances.1.1374AVANCES: INVESTIGACIÓN EN INGENIERÍA ISSN impreso: 1794-4953 ISSN online: 2619-6581 Vol. 15 (1) DOI: https://doi. org/10.18041/avances.v15i1. pp 131-149 (2018)

Bryson, J. (2004). What to do when stakeholders matter: stakeholder identification and analysis techniques. Public management review, 6(1), 21-53.

Calderón, M. (5 de mayo de 2016). Efectos de la dinámica urbana en los humedales de la ciudad de Bogotá: Diagnostico y propuestas de mejora en el marco del crecimiento verde. (Y. Cardozo, Entrevistador). 
Cerema. (2015). Milieux humides et aménagement urbain Dix expériences innovantes. Lyon: Recuperado de: http://es.calameo.com/ read/00009367920dcd9669f68.

EAAB. (2013). Ficha de inversión de Conservación y manejo ambiental. Bogotá: Recuperado de http:// www.acueducto.com.co/wpsv61/wps/html/resources/empresa/PeI/Macro7341-06172013.pdf.

EAAB. (29 de Enero de 2017). Empresa de Acueducto, agua, alcantarillado y aseo de Bogota. Obtenido de Acueducto y Alcantarillado de Bogotá: http://www.acueducto.com.co/wpsv61/wps/ portal/\%21ut/p/c5/hY5NDoIwEIXPwgHMDIW2sKyKbQ3QaAMWNoSFMSQCLoznF-JGTZR5y_9DNQwaWgf3aW9d-PQXsFBzZrIt5nliqDhZItaJEnEgvCoEj7xijUbKVTIUORDSkSSU3vAwgaog4X0ad57c0i6i1EbGqcmkz4iffF__TPHHyfwq_jg_1aMMjV2J-hWFipYnAMbr3DTq-kEJ

Escalona, A., \& González, G. (2016). Ensayo de modelización de la dinámica de los humedales de Bogotá como apoyo a su gestión ambiental en el marco del crecimiento verde. II congreso internacional; La ingeniería ambiental en el siglo XXI. Bogotá.

Organización de las Naciones Unidas para la Alimentación y la Agricultura-FAO. (1996). Desarrollo de Sistemas Agricolas y Conservacion del Suelo. Roma.

Franco, R. (2011). Tutorial arcgis. Recuperado de https://www.youtube.com/watch?v=00Zc7pJyigM \&list=PLAEhDYHUarGs3h5oQbWrXSbbb6yLVfhsv

ECOBARRIO LAGOS DE CASTILLA-González Angarita, G. (31. Recuperado de https://www.youtube.com/watch?v=fkPuoIwcCRA\&t=3s de Enero de 2018). Ecobarrio Lagos de Castilla. (H. González, Entrevistador)

Grimble, R., \& Wellard, K. (1997). Stakeholder methodologies in natural resource management: a review of principles, contexts, experiences and opportunities. Agricultural Systems s $55(2), 173-193$.
Instituto de Desarrollo Urbano-IDU. (2017). Inventario de vías de la ciudad de Bogotá. Bogotá. Recuperado de https://www.idu.gov.co/.

Inostroza, L., Baur, R., \& Csaplovics, E. (2013). Urban sprawl and fragmentation in Latin America: A dynamic quantification and characterization of spatial patterns. Journal of environmental management, $115,87-97$.

Lejano, R., Newbery, N., Ciolino, M., \& Newbery, D. (2019). Sustainability and incommensurability: Narrative policy analysis with application to urban ecology. Ecological economics. doi:10.1016/j. ecolecon.2019.06.003

Ricart, S., \& Clarimont, S. (2016). Modelling the links between irrigation, ecosystem services and rural development in pursuit of social legitimacy: Results from a territorial analysis of the Neste System (Hautes-Pyrénées, France). Journal of Rural Studies 43, 1-12.

Shlomo, A., Blei, A., Lamson-Hall, P., Galarza, N., Kumar, S., \& Shingade, S. (2018). Atlas de expanción urbana en Colombia. Bogotá. Obtenido de http://atlasexpansionurbanacolombia.org/ ciudades: NYU: Informe mundial sobre los asentamientos humanos.

Velez, I., Rátiva, S., \& Varela, D. (2012). Cartografía social como metodología participativa y colaborativa de investigación en el territorio. Cuadernos de geografía-Revista Colombiana de Geografía , 59-73.

Vásquez, A. (2016). Infraestructura verde, servicios ecosistémicos y sus aportes para enfrentar el cambio climático en ciudades: el caso del corredor ribereño del río Mapocho en Santiago de Chile. Revista de Geografía Norte Grande(63), 63-86.
Conflicto de Intereses Los autores declaran no tener ningún conflicto de intereses

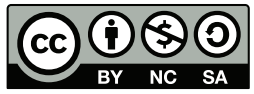


